MATHEMATICS OF COMPUTATION

Volume 79, Number 269, January 2010, Pages 603-612

S $0025-5718(09) 02288-1$

Article electronically published on July 8, 2009

\title{
ON EQUAL SUMS OF NINTH POWERS
}

\author{
A. BREMNER AND JEAN-JOËL DELORME
}

\begin{abstract}
In this paper, we develop an elementary method to obtain infinitely many solutions of the Diophantine equation

$$
x_{1}^{9}+x_{2}^{9}+x_{3}^{9}+x_{4}^{9}+x_{5}^{9}+x_{6}^{9}=y_{1}^{9}+y_{2}^{9}+y_{3}^{9}+y_{4}^{9}+y_{5}^{9}+y_{6}^{9}
$$
\end{abstract}

and we give some numerical results.

\section{INTRODUCTION}

Weisstein [7] gives a comprehensive survey of known results concerning equal sums of ninth powers. Moessner [4] in considering the Prouhet-Tarry-Escott problem gives a parametrization that inter alia gives infinitely many solutions to the Diophantine equation $\sum_{i=1}^{i=10} x_{i}^{9}=\sum_{i=1}^{i=10} y_{i}^{9}$. Only a few numerical solutions of the equation $\sum_{i=1}^{i=6} x_{i}^{9}=\sum_{i=1}^{i=6} y_{i}^{9}$ are known. Lander, Parkin, and Selfridge [2] give the single solution

$(23,18,14,13,13,1,22,21,15,10,9,5)$;

Ekl [1] lists eight other solutions, and Weisstein references nine more. A few additional solutions are listed in Piezas [5].

The solution in (1) satisfies the set of equalities:

$$
\left\{\begin{array}{c}
23+18+14+13+13+1=22+21+15+10+9+5 \\
23^{3}+18^{3}+14^{3}+13^{3}+13^{3}+1^{3}=22^{3}+21^{3}+15^{3}+10^{3}+9^{3}+5^{3} \\
23^{9}+18^{9}+14^{9}+13^{9}+13^{9}+1^{9}=22^{9}+21^{9}+15^{9}+10^{9}+9^{9}+5^{9}
\end{array}\right.
$$

Therefore this solution, as well as two other solutions on Ekl's list, satisfies the system $\left\{\left(\mathcal{T}_{1}\right),\left(\mathcal{T}_{3}\right),\left(\mathcal{T}_{9}\right)\right\}$, where $\left(\mathcal{T}_{p}\right)$ is the equality:

$$
\sum_{k=1}^{6} x_{k}^{p}=\sum_{k=1}^{6} y_{k}^{p}
$$

In this paper we actually prove that the system $\left\{\left(\mathcal{T}_{1}\right),\left(\mathcal{T}_{2}\right),\left(\mathcal{T}_{3}\right),\left(\mathcal{T}_{9}\right)\right\}$ has infinitely many rational solutions.

\section{A SIMPLER SYSTEM}

For any rational numbers $a_{1}, a_{2}, a_{3}, a_{1}^{\prime}, a_{2}^{\prime}, a_{3}^{\prime}$, we put

$$
\left\{\begin{array} { l } 
{ \sigma _ { 1 } = a _ { 1 } + a _ { 2 } + a _ { 3 } } \\
{ \sigma _ { 2 } = a _ { 2 } a _ { 3 } + a _ { 3 } a _ { 1 } + a _ { 1 } a _ { 2 } } \\
{ \sigma _ { 3 } = a _ { 1 } a _ { 2 } a _ { 3 } , }
\end{array} \quad \left\{\begin{array}{l}
\sigma_{1}^{\prime}=a_{1}^{\prime}+a_{2}^{\prime}+a_{3}^{\prime} \\
\sigma_{2}^{\prime}=a_{2}^{\prime} a_{3}^{\prime}+a_{3}^{\prime} a_{1}^{\prime}+a_{1}^{\prime} a_{2}^{\prime} \\
\sigma_{3}^{\prime}=a_{1}^{\prime} a_{2}^{\prime} a_{3}^{\prime} .
\end{array}\right.\right.
$$

Received by the editor March 3, 2009.

2000 Mathematics Subject Classification. Primary 11D41, 11 G05.

(C)2009 American Mathematical Society Reverts to public domain 28 years from publication 
Proposition 1. If the rational numbers $a_{1}, a_{2}, a_{3}, a_{1}^{\prime}, a_{2}^{\prime}, a_{3}^{\prime}$ satisfy the following system:

$$
(\mathcal{S}):\left\{\begin{array}{lll}
\left(\mathcal{S}_{1}\right): & \sigma_{1}=\sigma_{1}^{\prime} \\
\left(\mathcal{S}_{2}\right): & \sigma_{2}=\sigma_{2}^{\prime} \\
\left(\mathcal{S}_{3}\right): & 3 \sigma_{1}^{3}-3 \sigma_{1} \sigma_{2}+\sigma_{3}+\sigma_{3}^{\prime}=0,
\end{array}\right.
$$

then the rational numbers $x_{k}$ and $y_{k}, 1 \leq k \leq 6$, defined by the following set of equalities:

$$
\left\{\begin{array}{ccc}
x_{1}=a_{1}+\sigma_{1} & x_{2}=a_{2}+\sigma_{1} & x_{3}=a_{3}+\sigma_{1} \\
y_{1}=a_{1}-\sigma_{1} & y_{2}=a_{2}-\sigma_{1} & y_{3}=a_{3}-\sigma_{1} \\
& & \\
x_{4}=a_{1}^{\prime}-\sigma_{1}^{\prime} & x_{5}=a_{2}^{\prime}-\sigma_{1}^{\prime} & x_{6}=a_{3}^{\prime}-\sigma_{1}^{\prime} \\
y_{4}=a_{1}^{\prime}+\sigma_{1}^{\prime} & y_{5}=a_{2}^{\prime}+\sigma_{1}^{\prime} & y_{6}=a_{3}^{\prime}+\sigma_{1}^{\prime}
\end{array}\right.
$$

yield a solution of the following system:

(4)

$$
: \begin{cases}\left(\mathcal{T}_{1}\right): & x_{1}+x_{2}+x_{3}+x_{4}+x_{5}+x_{6}=y_{1}+y_{2}+y_{3}+y_{4}+y_{5}+y_{6}, \\ \left(\mathcal{T}_{2}\right): & x_{1}^{2}+x_{2}^{2}+x_{3}^{2}+x_{4}^{2}+x_{5}^{2}+x_{6}^{2}=y_{1}^{2}+y_{2}^{2}+y_{3}^{2}+y_{4}^{2}+y_{5}^{2}+y_{6}^{2}, \\ \left(\mathcal{T}_{3}\right): & x_{1}^{3}+x_{2}^{3}+x_{3}^{3}+x_{4}^{3}+x_{5}^{3}+x_{6}^{3}=y_{1}^{3}+y_{2}^{3}+y_{3}^{3}+y_{4}^{3}+y_{5}^{3}+y_{6}^{3}, \\ \left(\mathcal{T}_{9}\right): & x_{1}^{9}+x_{2}^{9}+x_{3}^{9}+x_{4}^{9}+x_{5}^{9}+x_{6}^{9}=y_{1}^{9}+y_{2}^{9}+y_{3}^{9}+y_{4}^{9}+y_{5}^{9}+y_{6}^{9} .\end{cases}
$$

Proof. First, make the assumption $\left(\mathcal{S}_{1}\right): \sigma_{1}=\sigma_{1}^{\prime}$. Then the system (3) immediately implies $\left(\mathcal{T}_{1}\right)$ and $\left(\mathcal{T}_{2}\right)$.

For any non-negative integer $p$, put $d_{p}=\sum_{k=1}^{3}\left(a_{k}^{p}-a_{k}^{\prime p}\right)$. Then

$$
\sum_{k=1}^{6}\left(x_{k}^{3}-y_{k}^{3}\right)=6 \sigma_{1} d_{2}
$$

and

$$
\sum_{k=1}^{6}\left(x_{k}^{9}-y_{k}^{9}\right)=6 \sigma_{1}\left(12 d_{2} \sigma_{1}^{6}+42 d_{4} \sigma_{1}^{4}+28 d_{6} \sigma_{1}^{2}+3 d_{8}\right) .
$$

The further assumption $\left(\mathcal{S}_{2}\right): \sigma_{2}=\sigma_{2}^{\prime}$ implies $d_{2}=0$, hence $\left(\mathcal{T}_{3}\right)$.

The assumptions $\left(\mathcal{S}_{1}\right),\left(\mathcal{S}_{2}\right)$ also imply the following:

$$
\left\{\begin{array}{l}
d_{4}=4\left(\sigma_{3}-\sigma_{3}^{\prime}\right) \sigma_{1} \\
d_{6}=3\left(\sigma_{3}-\sigma_{3}^{\prime}\right)\left(2 \sigma_{1}^{3}-4 \sigma_{1} \sigma_{2}+\sigma_{3}+\sigma_{3}^{\prime}\right) \\
d_{8}=4\left(\sigma_{3}-\sigma_{3}^{\prime}\right)\left(2 \sigma_{1}\left(\sigma_{1}^{2}-\sigma_{2}\right)\left(\sigma_{1}^{2}-3 \sigma_{2}\right)+\left(\sigma_{3}+\sigma_{3}^{\prime}\right)\left(3 \sigma_{1}^{2}-2 \sigma_{2}\right)\right) .
\end{array}\right.
$$

From this we deduce:

$$
\sum_{k=1}^{6}\left(x_{k}^{9}-y_{k}^{9}\right)=144 \sigma_{1}\left(\sigma_{3}-\sigma_{3}^{\prime}\right)\left(5 \sigma_{1}^{2}-\sigma_{2}\right)\left(3 \sigma_{1}^{3}-3 \sigma_{1} \sigma_{2}+\sigma_{3}+\sigma_{3}^{\prime}\right) .
$$

Consequently, from the assumption $\left(\mathcal{S}_{3}\right): 3 \sigma_{1}^{3}-3 \sigma_{1} \sigma_{2}+\sigma_{3}+\sigma_{3}^{\prime}=0$, we obtain $\left(\mathcal{T}_{9}\right)$. 
Remark 1. Equating to 0 other factors in (5) leads to trivial solutions.

For if $\sigma_{1}=0$, we obtain a trivial solution of $(\mathcal{T})$ satisfying $x_{i}=y_{i}, i=1, \ldots, 6$.

If $\sigma_{3}=\sigma_{3}^{\prime}$, then, taking into consideration the equalities $\sigma_{1}=\sigma_{1}^{\prime}$ and $\sigma_{2}=\sigma_{2}^{\prime}$, we deduce $a_{i}=a_{i}^{\prime}, i=1,2,3$, which leads to a trivial solution of $(\mathcal{T})$ satisfying

$$
\left(x_{1}, x_{2}, x_{3}, x_{4}, x_{5}, x_{6}\right)=\left(y_{4}, y_{5}, y_{6}, y_{1}, y_{2}, y_{3}\right) .
$$

If $5 \sigma_{1}^{2}-\sigma_{2}=0$, then necessarily $a_{1}, a_{2}, a_{3}$, and $a_{1}^{\prime}, a_{2}^{\prime}, a_{3}^{\prime}$ are zero, which again gives a trivial solution of $(\mathcal{T})$.

Remark 2. The sextuple $\left(a_{1}, a_{2}, a_{3}, a_{1}^{\prime}, a_{2}^{\prime}, a_{3}^{\prime}\right)=(17,-18,5,-19,9,14)$ is a solution of the system $(\mathcal{S})$ at (2), with $\sigma_{1}=4$. The numbers $x_{k}$ and $y_{k}$ from (3) give the following solution of the system $(\mathcal{T})$ at (4): $(21,-14,9,-23,5,10,13,-22,1,-15$, $13,18)$. This permutation of the solution at (1D) now satisfies the equation $\left(\mathcal{T}_{2}\right)$.

Henceforth we shall focus attention on the system $(\mathcal{S})$. Observe that if $\sigma_{1}=0$, then $\sigma_{1}^{\prime}=0$, and the system (3) shows that we obtain only trivial solutions of $(\mathcal{T})$, satisfying $x_{i}=y_{i}, i=1, \ldots, 6$. We shall refer to solutions of $(\mathcal{S})$ which satisfy $\sigma_{1}=0$ as trivial solutions of the system $(\mathcal{S})$.

Remark 3. The only solution of $(\mathcal{S})$ such that $a_{1}=a_{2}=a_{3}$ is the zero solution. For if in $(\mathcal{S})$ we replace $a_{2}$ and $a_{3}$ by $a_{1}$, we obtain:

$$
a_{1}^{\prime}+a_{2}^{\prime}+a_{3}^{\prime}=3 a_{1}, \quad a_{2}^{\prime} a_{3}^{\prime}+a_{3}^{\prime} a_{1}^{\prime}+a_{1}^{\prime} a_{2}^{\prime}=3 a_{1}^{2}, \quad a_{1}^{\prime} a_{2}^{\prime} a_{3}^{\prime}=-55 a_{1}^{3},
$$

so that the three numbers $a_{1}^{\prime}, a_{2}^{\prime}$ and $a_{3}^{\prime}$ are the solutions of the following equation: $x^{3}-3 a_{1} x^{2}+3 a_{1}^{2} x+55 a_{1}^{3}=0$. This may also be written as $\left(x-a_{1}\right)^{3}=-7\left(2 a_{1}\right)^{3}$. Since $x$ and $a_{1}$ are rational, we necessarily have $x=a_{1}$ and $a_{1}=0$, which implies $\left(a_{1}, a_{2}, a_{3}, a_{1}^{\prime}, a_{2}^{\prime}, a_{3}^{\prime}\right)=(0,0,0,0,0,0)$.

\section{AN ELLIPTIC SURFACE}

We first parametrize the two equations $\left(\mathcal{S}_{1}\right),\left(\mathcal{S}_{2}\right)$, at (2).

Proposition 2. Let $a=\left(a_{1}, a_{2}, a_{3}, a_{1}^{\prime}, a_{2}^{\prime}, a_{3}^{\prime}\right)$ be a sextuple of rational numbers. The following two statements are equivalent:

I: a satisfies the system: $\left\{\begin{array}{l}a_{1}+a_{2}+a_{3}=a_{1}^{\prime}+a_{2}^{\prime}+a_{3}^{\prime}, \\ a_{2} a_{3}+a_{3} a_{1}+a_{1} a_{2}=a_{2}^{\prime} a_{3}^{\prime}+a_{3}^{\prime} a_{1}^{\prime}+a_{1}^{\prime} a_{2}^{\prime} .\end{array}\right.$

II: There exist triples of rational numbers $p=\left(p_{1}, p_{2}, p_{3}\right)$ and $q=\left(q_{1}, q_{2}, q_{3}\right)$ such that

$$
p_{1}+p_{2}+p_{3}=0, \quad q_{1}+q_{2}+q_{3}=0
$$

and

$$
\left\{\begin{array} { l } 
{ a _ { 1 } - a _ { 1 } ^ { \prime } = p _ { 1 } q _ { 1 } } \\
{ a _ { 2 } - a _ { 2 } ^ { \prime } = p _ { 2 } q _ { 1 } } \\
{ a _ { 3 } - a _ { 3 } ^ { \prime } = p _ { 3 } q _ { 1 } , }
\end{array} \quad \left\{\begin{array} { l } 
{ a _ { 2 } - a _ { 3 } ^ { \prime } = p _ { 1 } q _ { 2 } } \\
{ a _ { 3 } - a _ { 1 } ^ { \prime } = p _ { 2 } q _ { 2 } } \\
{ a _ { 1 } - a _ { 2 } ^ { \prime } = p _ { 3 } q _ { 2 } , }
\end{array} \quad \left\{\begin{array}{l}
a_{3}-a_{2}^{\prime}=p_{1} q_{3} \\
a_{1}-a_{3}^{\prime}=p_{2} q_{3} \\
a_{2}-a_{1}^{\prime}=p_{3} q_{3} .
\end{array}\right.\right.\right.
$$

Proof. It is easily verified that II implies I. Let us prove that I implies II. For this purpose observe first that if $a_{1}+a_{2}+a_{3}=a_{1}^{\prime}+a_{2}^{\prime}+a_{3}^{\prime}$, then each of the following 
six numbers:

$$
\begin{array}{r}
\left(a_{1}-a_{1}^{\prime}\right)\left(a_{3}-a_{1}^{\prime}\right)-\left(a_{2}-a_{2}^{\prime}\right)\left(a_{2}-a_{3}^{\prime}\right) \\
\left(a_{2}-a_{2}^{\prime}\right)\left(a_{1}-a_{2}^{\prime}\right)-\left(a_{3}-a_{3}^{\prime}\right)\left(a_{3}-a_{1}^{\prime}\right) \\
\left(a_{3}-a_{3}^{\prime}\right)\left(a_{2}-a_{3}^{\prime}\right)-\left(a_{1}-a_{1}^{\prime}\right)\left(a_{1}-a_{2}^{\prime}\right) \\
-\left(a_{1}-a_{1}^{\prime}\right)\left(a_{1}-a_{3}^{\prime}\right)+\left(a_{2}-a_{2}^{\prime}\right)\left(a_{3}-a_{2}^{\prime}\right) \\
-\left(a_{2}-a_{2}^{\prime}\right)\left(a_{2}-a_{1}^{\prime}\right)+\left(a_{3}-a_{3}^{\prime}\right)\left(a_{1}-a_{3}^{\prime}\right) \\
-\left(a_{3}-a_{3}^{\prime}\right)\left(a_{3}-a_{2}^{\prime}\right)+\left(a_{1}-a_{1}^{\prime}\right)\left(a_{2}-a_{1}^{\prime}\right)
\end{array}
$$

is equal to $a_{2} a_{3}+a_{3} a_{1}+a_{1} a_{2}-\left(\begin{array}{llll}a_{2}^{\prime} & a_{3}^{\prime}+a_{3}^{\prime} & a_{1}^{\prime}+a_{1}^{\prime} & a_{2}^{\prime}\end{array}\right)$.

Consequently, if $a$ satisfies the two equalities in I, we obtain the six equalities:

$$
\begin{aligned}
& \left(a_{1}-a_{1}^{\prime}\right)\left(a_{3}-a_{1}^{\prime}\right)=\left(a_{2}-a_{2}^{\prime}\right)\left(a_{2}-a_{3}^{\prime}\right) \\
& \left(a_{2}-a_{2}^{\prime}\right)\left(a_{1}-a_{2}^{\prime}\right)=\left(a_{3}-a_{3}^{\prime}\right)\left(a_{3}-a_{1}^{\prime}\right) \\
& \left(a_{3}-a_{3}^{\prime}\right)\left(a_{2}-a_{3}^{\prime}\right)=\left(a_{1}-a_{1}^{\prime}\right)\left(a_{1}-a_{2}^{\prime}\right) \\
& \left(a_{1}-a_{1}^{\prime}\right)\left(a_{1}-a_{3}^{\prime}\right)=\left(a_{2}-a_{2}^{\prime}\right)\left(a_{3}-a_{2}^{\prime}\right) \\
& \left(a_{2}-a_{2}^{\prime}\right)\left(a_{2}-a_{1}^{\prime}\right)=\left(a_{3}-a_{3}^{\prime}\right)\left(a_{1}-a_{3}^{\prime}\right) \\
& \left(a_{3}-a_{3}^{\prime}\right)\left(a_{3}-a_{2}^{\prime}\right)=\left(a_{1}-a_{1}^{\prime}\right)\left(a_{2}-a_{1}^{\prime}\right) .
\end{aligned}
$$

Now consider the matrix:

$$
M=\left[\begin{array}{ccc}
a_{1}-a_{1}^{\prime} & a_{2}-a_{3}^{\prime} & a_{3}-a_{2}^{\prime} \\
a_{2}-a_{2}^{\prime} & a_{3}-a_{1}^{\prime} & a_{1}-a_{3}^{\prime} \\
a_{3}-a_{3}^{\prime} & a_{1}-a_{2}^{\prime} & a_{2}-a_{1}^{\prime}
\end{array}\right] .
$$

From the preceding six equalities, the rank of $M$ is 0 or 1 . As is well known, this implies the existence of two triples of rational numbers $p=\left(p_{1}, p_{2}, p_{3}\right)$ and $q=\left(q_{1}, q_{2}, q_{3}\right)$ such that

$$
\begin{array}{ccc}
a_{1}-a_{1}^{\prime}=p_{1} q_{1} & a_{2}-a_{3}^{\prime}=p_{1} q_{2} & a_{3}-a_{2}^{\prime}=p_{1} q_{3} \\
a_{2}-a_{2}^{\prime}=p_{2} q_{1} & a_{3}-a_{1}^{\prime}=p_{2} q_{2} & a_{1}-a_{3}^{\prime}=p_{2} q_{3} \\
a_{3}-a_{3}^{\prime}=p_{3} q_{1} & a_{1}-a_{2}^{\prime}=p_{3} q_{2} & a_{2}-a_{1}^{\prime}=p_{3} q_{3} .
\end{array}
$$

Since $a_{1}+a_{2}+a_{3}=a_{1}^{\prime}+a_{2}^{\prime}+a_{3}^{\prime}$, we obtain (6), (77), the equations defining II, as required.

Using the parametrization in (6), (17), of the equations $\left(\mathcal{S}_{1}\right),\left(\mathcal{S}_{2}\right)$, we make the following substitution into the equations $(\mathcal{S})$ :

$$
\begin{aligned}
a_{1}= & a_{3}^{\prime}+p_{2}\left(-q_{1}-q_{2}\right), \quad a_{2}=a_{3}^{\prime}+p_{1} q_{2}, & a_{3}=a_{3}^{\prime}+\left(-p_{1}-p_{2}\right) q_{1}, \\
& a_{1}^{\prime}=a_{3}^{\prime}+p_{2}\left(-q_{1}-q_{2}\right)-p_{1} q_{1}, & a_{2}^{\prime}=a_{3}^{\prime}+p_{1} q_{2}-p_{2} q_{1},
\end{aligned}
$$

where for the purpose of homogeneity we set $a_{3}^{\prime}=p_{2} q_{4}$, say. The third equation $\left(\mathcal{S}_{3}\right)$ delivers a homogeneous cubic equation in $\left\{q_{1}, q_{2}, q_{4}\right\}$, with coefficients homogeneous of degree 3 in $p_{1}, p_{2}$. Writing

$$
t=p_{1} / p_{2},
$$

this equation defines a curve of genus 1 over the function field $\mathbb{Q}(t)$, that is, an elliptic surface, and it is readily checked that the curve contains a point at 
$\left(q_{1}, q_{2}, q_{4}\right)=(1,1,1)$, so that the curve is actually elliptic over $\mathbb{Q}(t)$. Specifically, its equation has the form

$$
\begin{aligned}
C_{t} & :-3(2+t)\left(3+3 t+t^{2}\right) q_{1}^{3}+\left(-27-2 t+16 t^{2}+6 t^{3}\right) q_{1}^{2} q_{2}+7\left(11+11 t+3 t^{2}\right) q_{1}^{2} q_{4} \\
& -\left(15-16 t+2 t^{2}+6 t^{3}\right) q_{1} q_{2}^{2}-7\left(-11+5 t+5 t^{2}\right) q_{1} q_{2} q_{4}-56(2+t) q_{1} q_{4}^{2} \\
& +3(-1+t)\left(1-t+t^{2}\right) q_{2}^{3}+7\left(3-5 t+3 t^{2}\right) q_{2}^{2} q_{4}+56(-1+t) q_{2} q_{4}^{2}+56 q_{4}^{3}=0 .
\end{aligned}
$$

Points on the curve $C_{t}$ can be pulled back to solutions of the system $(\mathcal{T})$ by means of (3) and (8), namely:

$$
\begin{aligned}
& x_{1}: x_{2}: x_{3}: x_{4}: x_{5}: x_{6}: y_{1}: y_{2}: y_{3}: y_{4}: y_{5}: y_{6}= \\
&-(3+t) q_{1}+(-2+t) q_{2}+4 q_{4}:-(2+t) q_{1}+(-1+2 t) q_{2}+4 q_{4}: \\
&-(3+2 t) q_{1}+(-1+t) q_{2}+4 q_{4}: q_{1}-t q_{2}-2 q_{4}: \\
&(1+t) q_{1}+q_{2}-2 q_{4}:(2+t) q_{1}+(1-t) q_{2}-2 q_{4}: \\
&(1+t) q_{1}-t q_{2}-2 q_{4}:(2+t) q_{1}+q_{2}-2 q_{4}: \\
& q_{1}+(1-t) q_{2}-2 q_{4}:-(3+2 t) q_{1}+(-2+t) q_{2}+4 q_{4}: \\
&-(3+t) q_{1}+(-1+2 t) q_{2}+4 q_{4}:-(2+t) q_{1}+(-1+t) q_{2}+4 q_{4} .
\end{aligned}
$$

Symmetries of the solutions of $(\mathcal{S})$ under permutation of the $a_{i}, a_{i}^{\prime}$ induce symmetries of the underlying curve $C_{t}$, and the effect is that the curves corresponding to the parameters

$$
t,-1-\frac{1}{t},-\frac{1}{1+t},-\frac{t}{1+t},-1-t, \frac{1}{t}
$$

are isomorphic. A Weierstrass form for the elliptic curve $C_{t}$ is discovered to be the following:

$$
\begin{aligned}
E_{t}: Y^{2}= & X^{3}+1323\left(1+t+t^{2}\right)\left(23+69 t-54 t^{2}-223 t^{3}-54 t^{4}+69 t^{5}+23 t^{6}\right) X \\
& -2646\left(947+5682 t+16143 t^{2}+28630 t^{3}+22734 t^{4}-18342 t^{5}-46761 t^{6}\right. \\
& \left.-18342 t^{7}+22734 t^{8}+28630 t^{9}+16143 t^{10}+5682 t^{11}+947 t^{12}\right) .
\end{aligned}
$$

This latter curve has torsion group $\mathbb{Z} / 3 \mathbb{Z}$ over $\mathbb{Q}(t)$ with point of order 3 given by

$$
\left(147\left(1+t+t^{2}\right)^{2}, 756\left(1-t+t^{2}\right)\left(3+3 t+t^{2}\right)\left(1+3 t+3 t^{2}\right)\right),
$$

corresponding to the torsion point on $C_{t}$,

$$
T_{0}=(1,-2, t) \quad\left(\text { and where }-T_{0}=(2,-1,1+t)\right) .
$$

The condition that we derive a trivial solution of $(\mathcal{S})$ has become

$$
(t+2) q_{1}-(t-1) q_{2}-3 q_{4}=0,
$$

and this line cuts $C_{t}$ in precisely the three points of finite order. Thus only the torsion points of $C_{t}$ return trivial solutions of (2).

\section{Numerical RESUlts}

We can use standard computer software to determine values of the parameter $t$ for which the rational rank of the curve (11) is positive. The group of points on the associated curve $C_{t}$ is then infinite, and we are able to deduce an infinity of solutions to the system $(\mathcal{S})$ at (2), and hence an infinity of solutions to the system $(\mathcal{T})$ at (4). 
The effect of the symmetries is that it suffices to search with $1<t$, and in the range $3 \leq$ numerator $(t)+$ denominator $(t) \leq 20$ we obtain Table 1 , which lists the rational rank of (11) along with corresponding points $\left(q_{1}, q_{2}, q_{4}\right)$ on $C_{t}$. All computations were performed with Magma [3].

Example. We compute solutions of the system $(\mathcal{T})$ at (4) corresponding to $t=3$.

The curve $C_{3}$ has rank 1 with point of infinite order $Q=(1,8,-5)$, so that a point on $C_{3}$ is of type $n Q+\epsilon T_{0}, n \in \mathbb{Z}, \epsilon=0, \pm 1$. The pullbacks of the three points corresponding to $\epsilon=0, \pm 1$ give the same solution of the system (44) up to permutation, and so it is only necessary to consider $\epsilon=0$. Furthermore, the points $\pm n Q$ deliver the same solution up to permutation, and so we can further restrict ourselves to considering only the pullbacks of the points $n Q, n>0$. Table 2 lists the pullback points for $n=1, \ldots, 5$.

A similar chain of solutions can be computed for each of the $t$ corresponding to entries in Table 1, with a double infinity of solutions when the rank is 2 , and a triple infinity of solutions at $t=9 / 4$. We list some "small" solutions arising in this latter instance. Write $Q_{1}=(106,-282,279), Q_{2}=(124,-272,293), Q_{3}=$ $(239,-181,396)$ for the points from Table 1.

Note that the assertion at (10) is equivalent to the fact that the curves $C_{p_{i} / p_{j}}, 1 \leq$ $i \neq j \leq 3$, are isomorphic. The symmetry in the parametrization (7) interchanging $a_{2}^{\prime}$ and $a_{3}^{\prime}$ is equivalent to the symmetry interchanging $p_{i}$ with $q_{i}(i=1,2,3)$. Thus when $C_{p_{1} / p_{2}}$ has positive rank with point $\left(q_{1}, q_{2}, a_{3}^{\prime} / p_{2}\right)$, there is a corresponding point $\left(p_{1}, p_{2}, a_{2}^{\prime} / q_{2}\right)$ on $C_{q_{1} / q_{2}}$, and hence the isomorphic curves $C_{q_{i} / q_{j}}, 1 \leq i \neq j \leq$ 3 also have positive rank.

\section{Parametrizations over $\mathbb{Q}(\omega)$}

It would be useful to know the $\mathbb{Q}(t)$ rank of the curve $E_{t}$ at (11). If this were positive, then corresponding points on $C_{t}$ would pull back to solutions of the system $(\mathcal{T})$ that are polynomials in $\mathbb{Z}[t]$. Unfortunately, we are unable to determine whether or not $E_{t}$ contains any non-torsion points over $\mathbb{Q}(t)$. The large points in Table 1 for $t=15 / 2,12 / 7,11 / 9$, together with several specializations of $E_{t}$ that have rank 0 , would suggest that the parametrization of a point is unlikely, and therefore that the $\mathbb{Q}(t)$-rank is 0 . The equation $E_{t}$ is that of an elliptic $K 3$-surface, and as such, methods of Shioda [6] allow us to compute an upper bound for the rank of $E_{t}$ over $\mathbb{C}(t)$, and this turns out to be 3 . We are able to spot two independent points $P_{1}$, $P_{2}$ of infinite order on $C_{t}$ over $\mathbb{C}(t)$, but have not determined whether or not there is a third:

$$
P_{1}=(6,-4-2 \omega, 3+3 t), \quad P_{2}=\left(-\omega, 1, \frac{1}{2}(1-\omega)\right),
$$

where $\omega=\frac{1}{2}(-1+\sqrt{-3})$ is a complex cube root of unity. Summing each point with its Galois conjugate:

$$
P_{1}+\bar{P}_{1}=(1,-2, t), \quad P_{2}+\bar{P}_{2}=(1,1,1),
$$

we obtain points of finite order. Thus no non-trivial rational polynomial parametrization of $(\mathcal{T})$ arises in this way. The two points $P_{1}, P_{2}$ do generate infinitely many polynomial parametrizations of $(\mathcal{T})$, but of course with coefficients over $\mathbb{Q}(\omega)$. For example, the pullbacks of $P_{1}$ and $P_{2}$ result in trivial solutions of the system (2); 
TABLE 1. Values of $t$ with $C_{t}$ of positive rank

\begin{tabular}{|c|c|c|}
\hline$t$ & independent point(s) on $C_{t}$ & rank \\
\hline 3 & $(1,8,-5)$ & 1 \\
\hline 4 & $(1446,14711,-15299)$ & 1 \\
\hline $5 / 2$ & $(356,1882,-647)$ & 1 \\
\hline $4 / 3$ & $(25,278,-12)$ & 1 \\
\hline 7 & $(207,-52,785)$ & 1 \\
\hline 8 & $(1,3,-5)$ & 1 \\
\hline $7 / 2$ & $(82,-664,821)$ & 1 \\
\hline $5 / 4$ & $(2648,539468,-54847)$ & 1 \\
\hline $7 / 3$ & $(543,1656,-125),(9137158,2514571,13080130)$ & 2 \\
\hline 10 & $(59616,83123,-33345)$ & 1 \\
\hline $6 / 5$ & $(6549648283004459,-22665359828265916,8749022109376884)$ & 1 \\
\hline 11 & $(479,-2916,13421),(14033,-43851,222497)$ & 2 \\
\hline 12 & $(19,64,-190)$ & 1 \\
\hline $11 / 2$ & $(-27783615238,-228089845712,349090862393)$ & 1 \\
\hline $10 / 3$ & $(94087753,101830636,85790044)$ & 1 \\
\hline $9 / 4$ & $(106,-282,279),(124,-272,293),(239,-181,396)$ & 3 \\
\hline $8 / 5$ & $(36194817,128001892,11175862)$ & 1 \\
\hline $7 / 6$ & $(3049,-8401,3738),(35004,-61398,40157)$, & 2 \\
\hline 13 & $(4724,1055987,-5026528)$ & 1 \\
\hline $9 / 5$ & $(121419260,-1377907645,613796368)$ & 1 \\
\hline $11 / 4$ & $(126758321,-30905647,231572729)$ & 1 \\
\hline $8 / 7$ & $\begin{array}{l}(14818297849097,12821985982426,14953926106708) \\
\end{array}$ & 1 \\
\hline $13 / 3$ & $(150351,100917,224711),(13051968,-12150687,37914218)$ & 2 \\
\hline $11 / 5$ & $(154090,443445,-3052)$ & 1 \\
\hline $9 / 7$ & $(742,-2709,1116),(2455971,3614233,2299483)$ & 2 \\
\hline 16 & $(18,179,-963),(958,6731,-34507)$ & 2 \\
\hline $15 / 2$ & $\begin{array}{c}134207609048965376073388156958141987, \\
28950557364798896116286088178331879, \\
422719433838369207424332656027729381)\end{array}$ & 1 \\
\hline $14 / 3$ & $(359601,464652,185656),(12153,-8250,35330)$ & 2 \\
\hline $13 / 4$ & $(25702,74362,-22943)$ & 1 \\
\hline $11 / 6$ & $(1532,-16154,7563)$ & 1 \\
\hline $9 / 8$ & $\begin{array}{c}(27787,-37949,30240),(21756703708,-959147282732,73870303329) \\
\end{array}$ & 2 \\
\hline $11 / 7$ & $(11754403807181,-239314804762243,71755024964941)$ & 1 \\
\hline 18 & $(7901044,-22041517,187889479)$ & 1 \\
\hline $17 / 2$ & $(153,599,-1262),(1874,2748,-1015)$ & 2 \\
\hline $15 / 4$ & $(4688,8548,-97),(880834,-4824818,6950465)$ & 2 \\
\hline $14 / 5$ & $(23,108,-44),(1287580662737,1391088002287,1201351021327)$ & $\overline{2}$ \\
\hline $13 / 6$ & $\begin{array}{l}(4093367511,4633565061,3798630686) \\
\end{array}$ & 1 \\
\hline $12 / 7$ & $\begin{array}{l}4607882348502503129769514, \\
7355497055510069300923039, \\
3688741999490895912106389)\end{array}$ & 1 \\
\hline $11 / 8$ & $\begin{array}{l}(2720488,6913952,1992365) \\
\end{array}$ & 1 \\
\hline $10 / 9$ & $\begin{array}{l}(343956623832196,-126385184284523,362777970896793) \\
\end{array}$ & 1 \\
\hline $17 / 3$ & $(127,-596,1405)$ & 1 \\
\hline $11 / 9$ & $\begin{array}{c}\text { (888221912602727881028999424599, } \\
\text {-492896695539027689262279545737, } \\
987459994593608883597631915735)\end{array}$ & 1 \\
\hline
\end{tabular}


TABLE 2. Solutions to the system (41) arising from $t=3$

\begin{tabular}{c||c|c|c|c|c|}
$n$ & 1 & 2 & 3 & 4 & 5 \\
\hline$x_{1}$ & 18 & 13825 & -176607027 & -295503813128476 & 16781116061381923831545 \\
$x_{2}$ & -15 & -9157 & 154786818 & 163694336414557 & 7388891819632091442261 \\
$x_{3}$ & 13 & 1092 & 134510017 & 453392718089919 & -21580737831236008171454 \\
$x_{4}$ & 13 & 4495 & -22242470 & 129024868182881 & -17757024654750594094103 \\
$x_{5}$ & -22 & -14187 & 168678401 & 198009483059676 & -4986911769004750233739 \\
$x_{6}$ & 1 & 6812 & -202780835 & -487825971930557 & 21449301398866340776666 \\
$y_{1}$ & 10 & 10945 & -232951931 & -456295433816476 & 15486481036492920280369 \\
$y_{2}$ & -23 & -12037 & 98441914 & 2902715726557 & 6094256794743087891085 \\
$y_{3}$ & 5 & -1788 & 78165113 & 292601097401919 & -22875372856125011722630 \\
$y_{4}$ & 21 & 7375 & 34102434 & 289816488870881 & -16462389629861590542927 \\
$y_{5}$ & -14 & -11307 & 225023305 & 358801103747676 & -3692276744115746682563 \\
$y_{6}$ & 9 & 9692 & -146435931 & -327034351242557 & 22743936423755344327842 \\
\hline & \multicolumn{5}{|l}{}
\end{tabular}

TABLE 3. Solutions to the system (4) arising from the rank 3 curve

\begin{tabular}{|c|c|c|c|c|c|c|}
\hline point on $C_{9 / 4}$ & $\begin{array}{l}x_{1} \\
y_{1}\end{array}$ & $\begin{array}{l}x_{2} \\
y_{2}\end{array}$ & $\begin{array}{l}x_{3} \\
y_{3}\end{array}$ & $\begin{array}{l}x_{4} \\
y_{4}\end{array}$ & $\begin{array}{l}x_{5} \\
y_{5}\end{array}$ & $\begin{array}{l}x_{6} \\
y_{6}\end{array}$ \\
\hline \multirow[b]{2}{*}{$Q_{2}$} & 453 & 150 & -307 & -455 & -98 & 281 \\
\hline & 429 & 174 & -331 & -431 & -122 & 305 \\
\hline \multirow[b]{2}{*}{$Q_{1}$} & 978 & 365 & -643 & -991 & -63 & 490 \\
\hline & 842 & 501 & -779 & -855 & -199 & 626 \\
\hline \multirow[b]{2}{*}{$Q_{3}$} & -1136 & 583 & 261 & 785 & 1739 & -1800 \\
\hline & -1568 & 1015 & -171 & 1217 & 1307 & -1368 \\
\hline \multirow[b]{2}{*}{$Q_{1}+Q_{2}$} & -3147 & 2120 & 75 & 1927 & 4832 & -4927 \\
\hline & -4027 & 3000 & -805 & 2807 & 3952 & -4047 \\
\hline \multirow[b]{2}{*}{$Q_{1}+Q_{3}$} & 6190 & -18943 & 17613 & 3265 & -12363 & 9958 \\
\hline & 470 & -13223 & 11893 & 8985 & -18083 & 15678 \\
\hline \multirow[b]{2}{*}{$Q_{1}+Q_{2}+Q_{3}$} & 159999 & 99268 & -117995 & -175511 & 67884 & 21299 \\
\hline & 105055 & 154212 & -172939 & -120567 & 12940 & 76243 \\
\hline \multirow[b]{2}{*}{$Q_{2}-Q_{3}$} & 319333 & -455078 & 333993 & -72523 & -428926 & 415401 \\
\hline & 207133 & -342878 & 221793 & 39677 & -541126 & 527601 \\
\hline
\end{tabular}

however, the pullbacks of $2 P_{1}$ and $2 P_{2}$ result in the following cubic parametrizations, respectively:

$$
\begin{array}{r}
x_{1}: x_{2}: x_{3}: x_{4}: x_{5}: x_{6}: y_{1}: y_{2}: y_{3}: y_{4}: y_{5}: y_{6}= \\
(3 \omega+3) t^{3}+(\omega+4) t^{2}+(-\omega+3) t-3 \omega: \\
(-2 \omega-1) t^{3}+(3 \omega+3) t^{2}+(4 \omega+4) t+\omega+2: \\
3 \omega t^{3}+(2 \omega-1) t^{2}+(-9 \omega-6) t-2 \omega-1: \\
(-\omega-2) t^{3}+(-5 \omega-6) t^{2}+(5 \omega-1) t+\omega-1: \\
(2 \omega+1) t^{3}+(9 \omega+3) t^{2}+(-2 \omega-3) t-3 \omega-3:
\end{array}
$$




$$
\begin{array}{r}
(-\omega+1) t^{3}-4 \omega t^{2}-3 \omega t+2 \omega+1: \\
(-\omega+1) t^{3}+(-5 \omega+1) t^{2}+(5 \omega+6) t+\omega+2: \\
(2 \omega+1) t^{3}+(9 \omega+6) t^{2}+(-2 \omega+1) t-3 \omega: \\
(-\omega-2) t^{3}+(-4 \omega-4) t^{2}+(-3 \omega-3) t+2 \omega+1: \\
3 \omega t^{3}+(\omega-3) t^{2}+(-\omega-4) t-3 \omega-3: \\
(-2 \omega-1) t^{3}+3 \omega t^{2}+4 \omega t+\omega-1: \\
(3 \omega+3) t^{3}+(2 \omega+3) t^{2}+(-9 \omega-3) t-2 \omega-1 ;
\end{array}
$$

and

$$
\begin{array}{r}
x_{1}: x_{2}: x_{3}: x_{4}: x_{5}: x_{6}: y_{1}: y_{2}: y_{3}: y_{4}: y_{5}: y_{6}= \\
(2 \omega+4) t^{3}+(4 \omega+6) t^{2}+(5 \omega+8) t+3 \omega+3: \\
-2 t^{3}+(3 \omega+2) t^{2}+(3 \omega+1) t+\omega-1: \\
(-4 \omega-6) t^{3}+(-4 \omega-4) t^{2}+(\omega+7) t+3: \\
(-2 \omega-2) t^{3}+(-5 \omega-8) t^{2}+(-6 \omega-9) t-\omega-2: \\
(-2 \omega-6) t^{3}+(-6 \omega-14) t^{2}-3 t+\omega+2: \\
(4 \omega+8) t^{3}+(5 \omega+12) t^{2}+2 t-2 \omega-1: \\
(4 \omega+8) t^{3}+(7 \omega+12) t^{2}+(2 \omega+2) t+\omega-1: \\
(-2 \omega-6) t^{3}-4 t^{2}+(6 \omega+7) t+3 \omega+3: \\
(-2 \omega-2) t^{3}+(-\omega+2) t^{2}+(-2 \omega+1) t-2 \omega-1: \\
(-4 \omega-6) t^{3}+(-8 \omega-14) t^{2}+(-3 \omega-3) t+\omega+2: \\
-2 t^{3}+(-3 \omega-8) t^{2}+(-3 \omega-9) t-\omega-2: \\
(2 \omega+4) t^{3}+(2 \omega+6) t^{2}+(3 \omega+8) t+3 .
\end{array}
$$

\section{ACKNOWLEDGEMENTS}

The second author warmly thanks Dr. Simon J. Agou for his kindness and encouragement, and Dr. François Vellutini and Yves Mannheimer for reading an initial version of the manuscript.

\section{REFERENCES}

[1] R. EkL, New results in equal sums of like powers, Math. Comp. 67, no. 223 (1998), 1309-1315. MR.1474650 (98m:11023)

[2] L.J. Lander, T.R. Parkin, and J.L. Selfridge, A survey of equal sums of like powers, Math. Comp. 21 (1967), 446-459. MR.0222008 (36:5060)

[3] W. Bosma, J. Cannon, And C. Playoust, The Magma algebra system. I. The user language. J. Symbolic Comput., 24(3-4): 235-265, 1997. MR1484478

[4] A. Moessner, On Equal Sums of Like Powers, Math. Student 15 (1947), 83-88. MR0033840 (11:500a)

[5] Titus Piezas III, Timeline of Euler's Extended Conjecture, http://www.geocities.com/ titus_piezas/Timeline1.htm.

[6] T. ShiodA, On elliptic modular surfaces, J. Math. Soc. Japan, 24 (1972) 20-59. MR0429918 $(55: 2927)$ 
[7] E.W. Weisstein, Diophantine Equation-9th Powers, http://mathworld.wolfram.com/ DiophantineEquation9thPowers.html

Department of Mathematics, Arizona State University, Tempe, Arizona

E-mail address: bremner@asu.edu

$U R L:$ http://math.asu.edu/ andrew/

6 RUe Des Émeraudes, 69006 Lyon, France

E-mail address: jean.joel.delorme@wanadoo.fr 